\title{
Feedback Stabilization of High-Speed Planing Vessels by a Controllable Transom Flap
}

\author{
Handa Xi, Student Member, IEEE, and Jing Sun, Fellow, IEEE
}

\begin{abstract}
This paper focuses on the mitigation of porpoising instability of high-speed planing vessels using controllable transom flap and dynamic feedback. A control oriented model that captures both steady-state and dynamic characteristics is presented and used to facilitate the model-based control design. A nonlinear controller is developed based on the feedback linearization method to achieve asymptotic stability of the planing boat, thus avoiding porpoising at high speeds. We first show that the full-state nonlinear dynamic model describing the ship motion is not feedback linearizable. A state transformation is then constructed to decompose the model into a linearizable subsystem and a nonlinear internal dynamic subsystem. A reduced order state feedback is shown next to stabilize the planing vessel motion around the equilibrium point. Analysis of the region of attraction is also performed to provide an assessment of the effective safe operating range around the equilibrium point.
\end{abstract}

Index Terms-Feedback control, planing boat, porpoising, stabilization.

\section{INTRODUCTION}

A HIGH-SPEED planing vessel has a substantial portion of its weight supported by the hydrodynamic lift, in contrast to the conventional displacement vessel which is supported primarily by the hydrostatic buoyancy force. Due to the complicated nature of the hydrodynamic forces, high-speed planing boats face dynamic instability problems in both vertical and transverse planes, such as porpoising, chine walking, progressive heeling, unstable pitching-induced rolling, or a combination of these motions [1], [2].

Porpoising might be the most well-known instability phenomenon of the high-speed planing craft. It refers to the periodic, coupled heave/pitch oscillation in the vertical plane that a planing vessel may experience at high speeds. The motion is sustained by the energy derived from the craft's forward speed and the planing lift force.

The study of the vertical-plane motion of high-speed planing vessels can be traced back to the early twentieth century, and the research became a very active and fruitful field during 1960s-1990s [3]-[8]. These earlier works focused mainly on the effects of design parameters, such as the location of the center of gravity, load, forward speed, and other geometric parameters of the planing hull, on the characteristics of the craft's motion.

Manuscript received April 04, 2005; accepted November 25, 2005. Associate Editor: H. Maeda.

The authors are with the Naval Architecture and Marine Engineering Department, University of Michigan, Ann Arbor, MI 48109 USA (e-mail: jingsun@umich.edu).

Digital Object Identifier 10.1109/JOE.2006.875097
While the research community was focusing on understanding the fundamentals of the dynamics of high-speed planing boats, scientists and engineers had introduced appendages to control the vertical-plane motion of high-speed vessels. In [9], controllable transom flaps and T-foils were adopted to reduce motion sickness aboard a high-speed ferry. Savitsky and Brown [10] studied the hydrodynamic force induced by a static transom flap and its effects on the running trim, drag, power requirement, and porpoising stability of the planing hull. Compared to the substantial research progress on the hydrodynamics and design of the high-speed planing craft, there has been little work published on the vertical-plane motion control for planing boats using controllable appendages.

Recently, a control-oriented nonlinear model has been developed by the authors for high-speed prismatic planing vessels equipped with controllable transom flaps [11]. The effects of preset static deflections of the transom flap on the running attitude and motion characteristics of planing boats are analyzed. In this paper, we investigate the feedback stabilization problem for the planing vessels using nonlinear control theory based on feedback linearization. A state transformation is constructed to transform the system into a partially feedback linearizable form, as it is demonstrated that the system is not fully feedback linearizable. Local asymptotic stability is obtained by designing a stabilizing feedback control for the linear subsystem and verifying the local stability of the nonlinear zero dynamics. With analysis of the internal dynamics, we prove that motion stability of the high-speed planing vessel can be guaranteed by the proposed controller for initial conditions from which the boat's motion trajectory remains in the applicable range of Savitsky's methods.

The organization of this paper is as follows. In Section II, a nonlinear model is described for the prismatic planing vessel with a controllable transom flap. The open loop stability of the planing vessel with a fixed static deflection of the transom flap is analyzed in Section III. In Section IV, a stabilizing controller, based on the feedback linearization method, is designed to maintain the boat's stability at high speeds. The region of attraction is analyzed in Section $\mathrm{V}$, before concluding remarks are given in Section VI.

\section{NONLINEAR MODEl OF Planing Vessels With CONTROLlable TRANSOM FlaPS}

The model described in this section combines fundamental physical laws and empirical relations. It provides the necessary tool for system analysis and controller design using modelbased methodologies. 


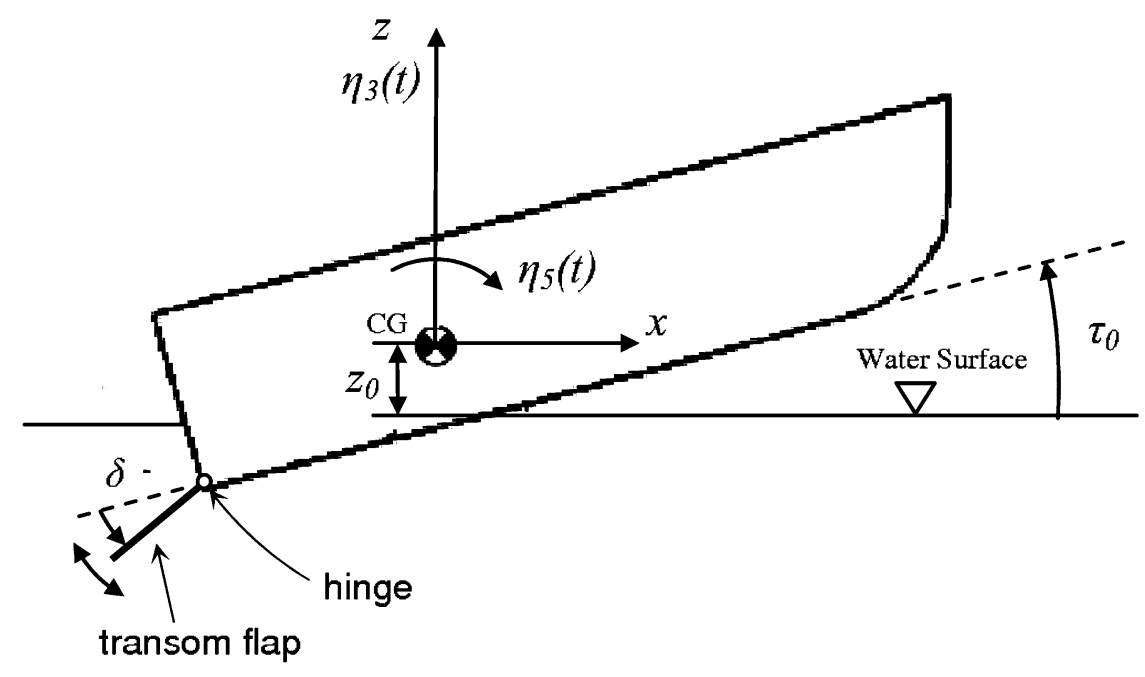

Fig. 1. Coordinate system of the planing vessel.

\section{A. Coordinate System and Motion Equation}

A right-handed coordinate system is defined in Fig. 1 for the prismatic planing vessel running in calm water. At the equilibrium running attitude, the trim angle is defined as $\tau_{0}$, and the vertical distance of the center of gravity (CG) from the water level as $z_{0} . \eta_{3}(t)$ and $\eta_{5}(t)$ are the vertical displacement (i.e., heave) of CG and the rotation (i.e., pitch) of the vessel relative to the inertia axis, respectively. $\eta_{3}(t)$ is positive upward and $\eta_{5}(t)$ positive bow down, as shown in Fig. 1. The notations and directions are chosen to be consistent with those used in [4] and [5]. $\eta_{3}(t)$ and $\eta_{5}(t)$ can be expressed as follows, respectively:

$$
\begin{aligned}
& \eta_{3}(t)=z(t)-z_{0} \\
& \eta_{5}(t)=-\left(\tau(t)-\tau_{0}\right)
\end{aligned}
$$

where $\tau(t)$ and $z(t)$ are the effective trim angle and the effective vertical distance of CG from the water level of the craft in motion, respectively.

For the vertical-plane motion of the planing craft, it is generally accepted that the heave/pitch motion can be decoupled from the surge motion for small trim angles [6], [7]. This is the case focused on in this paper, and consequently, only heave/pitch motions will be considered.

By adding the forces induced by the transom flap to the form used in [4] and [5], the motion equation of the planing vessel with a controllable transom flap running in the calm water can be written as follows:

$$
A \ddot{\eta}+B \dot{\eta}=F^{R}+F^{F}
$$

where

$$
\begin{aligned}
\eta & =\left[\begin{array}{l}
\eta_{3} \\
\eta_{5}
\end{array}\right] \quad F^{R}=\left[\begin{array}{c}
F_{3}^{R} \\
F_{5}^{R}
\end{array}\right] \quad F^{F}=\left[\begin{array}{l}
F_{3}^{F} \\
F_{5}^{F}
\end{array}\right] \\
A & =\left[\begin{array}{cc}
m+a_{33} & a_{35} \\
a_{53} & I_{55}+a_{55}
\end{array}\right] \quad B=\left[\begin{array}{ll}
b_{33} & b_{35} \\
b_{53} & b_{55}
\end{array}\right] .
\end{aligned}
$$

$m$ is the vessel mass and $I_{55}$ the pitch moment of inertia about CG. $a_{i j}$ and $b_{i j}, i, j=3,5$ are the added mass and damping coefficients, respectively. $F_{3}^{R}$ and $F_{5}^{R}$ are the heave and pitch restoring forces, respectively. $F_{3}^{F}$ and $F_{5}^{F}$ are the forces induced by the transom flap in heave and pitch direction, respectively.

To obtain a model of the planing vessel, those coefficients and forces need to be determined. In our work, $A$ and $B$ are determined based on experimental results of [4], and $F^{R}$ and $F^{F}$ are calculated using Savitsky's method [3], [10].

\section{B. Added Mass and Damping Coefficients}

Experiments have shown that the added mass $a_{i j}$ and damping coefficients $b_{i j}$ for prismatic planing vessels are nonlinear functions of the motion amplitude and frequency [4]. However, compared to the nonlinearities of the restoring forces which will be discussed later, the effects of nonlinearities in $a_{i j}$ and $b_{i j}$ on the craft's motion are small [5]. Therefore, $A$ and $B$ are assumed to be constant at a given forward speed and running attitude. Their values are extrapolated from the experimental results in [4], depending upon the speed, the equilibrium trim angle, and mean wetted length beam ratio.

\section{Restoring Forces}

The forces acting on the planing hull with a transom flap are shown in Fig. 2, where $N$ is the water pressure acting normal to the bottom, $T$ the thrust force, $D_{f}$ the frictional drag, $F$ the hydrodynamic force acting on the transom flap, $\varepsilon$ the inclination of the thrust relative to the keel, $l_{p}$ the longitudinal distance of the center of pressure measured from the transom, $a$ the distance between $D_{f}$ and CG, $f$ the distance between $T$ and CG, $\delta$ the deflection of the transom flap, and $g$ the gravity acceleration.

From Fig. 2, the restoring forces can be expressed as follows:

$$
\begin{aligned}
F_{3}^{R} & =N \cos \tau+T \sin (\tau+\epsilon)-D_{f} \sin \tau-\mathrm{mg} \\
F_{5}^{R} & =D_{f} a-N\left(l_{p}-l_{\mathrm{cg}}\right)-T f \\
\tau(t) & =\tau_{0}-\eta_{5}(t) .
\end{aligned}
$$

Consider the simpler case studied in [3] where the thrust and the frictional drag pass through CG, i.e., $a=f=0$. To simplify the model, it is also assumed that $\varepsilon=0$, and the sine terms in 


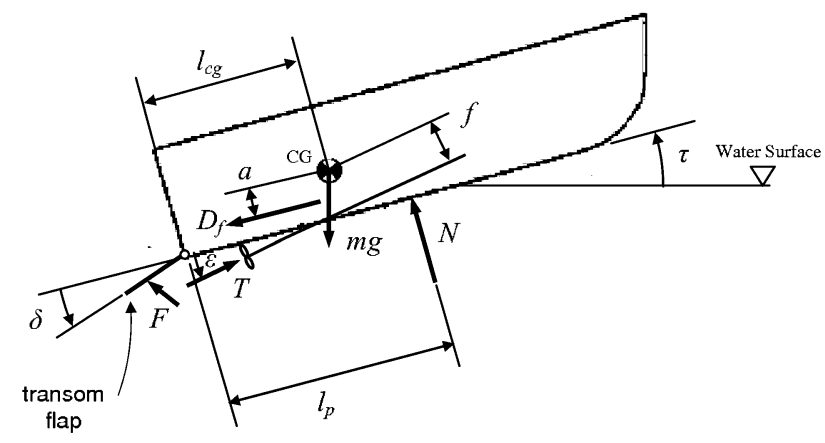

Fig. 2. Forces acting on the planing hull with a transom flap.

the right hand side of (4) are neglected for small trim angles. Hence, the restoring forces become

$$
\begin{aligned}
& F_{3}^{R}=N \cos \left(\tau_{0}-\eta_{5}\right)-\mathrm{mg} \\
& F_{5}^{R}=-N\left(l_{p}-l_{\mathrm{cg}}\right) .
\end{aligned}
$$

As mentioned in [4], it is supposed that the effects of the acceleration and velocity of the craft's motion on the hydrodynamic forces have been captured in terms of added mass and damping coefficients in the left hand side of (3). Therefore, only those components as functions of the craft's displacement are considered to determine $F^{R}$.

Savitsky [3] gave empirical formulas to calculate the restoring forces as functions of the effective mean wetted length beam ratio $\lambda$ and the effective trim angle $\tau$. The involved formulas are listed as follows.

$$
\begin{aligned}
C_{L_{0}} & =\tau^{1.1}\left(0.0120 \lambda^{0.5}+\frac{0.0055 \lambda^{2.5}}{C_{v}^{2}}\right) \\
C_{L_{\beta}} & =C_{L_{0}}-0.0065 \beta C_{L_{0}}^{0.6} \\
N & =\frac{0.5 \rho V^{2} B^{2} C_{L_{\beta}}}{\cos \tau} \\
l_{p} & =\lambda B\left(0.75-\frac{1}{5.21 \frac{C_{v}^{2}}{\lambda^{2}}+2.39}\right)
\end{aligned}
$$

where $C_{v}=V / \sqrt{g B}$ is the forward speed coefficient, $V$ the forward speed, $B$ the beamlength, $C_{L_{0}}$ lift coefficient for a zero deadrise surface, $C_{L_{\beta}}$ lift coefficient for surface with constant deadrise of $\beta$, and $\rho$ water density.

To express the restoring forces as explicit functions of craft displacements, the following relation developed in [4] is adopted to describe $\lambda$ as a function of $\left(z_{0}, \tau_{0} ; \eta_{3}, \eta_{5}\right)$ :

$$
\lambda(t)=\frac{1}{B}\left[l_{\mathrm{cg}}+\frac{v_{\mathrm{cg}}}{\tan \left(\tau_{0}-\eta_{5}(t)\right)}-\frac{z_{0}+\eta_{3}(t)}{\sin \left(\tau_{0}-\eta_{5}(t)\right)}\right]
$$

where $v_{\mathrm{cg}}$ is the vertical distance of CG from the keel.

Given the speed $V$, the equilibrium running attitude $\left(z_{0}, \tau_{0}\right)$, and the motion displacement $\left(\eta_{3}(t), \eta_{5}(t)\right)$, the restoring forces $F^{R}(t)$ can be obtained by (7)-(13).

\section{Forces by Controllable Transom Flap}

Savitsky and Brown [10] provided empirical formulas to calculate the forces induced by the transom flap as linear functions of the flap deflection $\delta$, which is viewed as the control input to the system. The flap lift $F_{3}^{F}$, and the flap moment about the boat $\mathrm{CG}, F_{5}^{F}$, can be determined by (14) and (15), respectively

$$
\begin{aligned}
& F_{3}^{F}=d_{3} \delta:=0.023 L_{F} B \sigma \rho V^{2} \delta \\
& F_{5}^{F}=d_{5} \delta:=d_{3}\left[l_{\mathrm{cg}}-0.6 B-L_{F}(1-\sigma)\right] \delta
\end{aligned}
$$

where $d_{3}, d_{5}$ are constant coefficients, $L_{F}$ the flap chord length, and $\sigma$ the flap span-beam ratio. $B, \rho$, and $V$ are defined as in (9)-(12). Note that, by following Savitsky and Brown's results, we only consider the force caused by the flap deflection. From results in aerodynamics, it has been found that forces induced by the wing flap can be also dependent on the velocity and acceleration of the flap motion [14]. However, the relationship of these force components for high-speed planing hulls could not be found in the open literature and, therefore, have not been incorporated in the model developed in this paper.

To summarize, the motion equation of the high-speed planing vessel with a controllable transom flap running in calm water becomes

$$
A \ddot{\eta}+B \dot{\eta}=F^{R}\left(z_{0}, \tau_{0} ; \eta\right)+F^{F}(\delta)
$$

where $F^{R}\left(z_{0}, \tau_{0} ; \eta\right)$ is determined by (7)-(13) and $F^{F}(\delta)$ by (14) and (15).

By defining the state vector $x=\left[x_{1}, x_{2}, x_{3}, x_{4}\right]^{T}=\left[\eta_{3}, \eta_{5}\right.$, $\left.\dot{\eta}_{3}, \dot{\eta}_{5}\right]^{T}$, the motion equation can be transformed to the statespace form as follows:

$$
\dot{x}=f\left(z_{0}, \tau_{0} ; x\right)+b \delta
$$

where

$$
\begin{aligned}
f\left(z_{0}, \tau_{0} ; x\right) & =\left[\begin{array}{cc}
0_{2 \times 2} & I_{2 \times 2} \\
0_{2 \times 2} & -A^{-1} B
\end{array}\right] x+\left[\begin{array}{c}
0_{2 \times 1} \\
A^{-1} F^{R}\left(z_{0}, \tau_{0} ; x\right)
\end{array}\right] \\
b & =\left[\begin{array}{c}
0_{2 \times 1} \\
A^{-1}\left[\begin{array}{l}
d_{3} \\
d_{5}
\end{array}\right]
\end{array}\right] .
\end{aligned}
$$

In general, the restoring forces $F^{R}$ are nonlinear functions of the motion displacement $\left(\eta_{3}, \eta_{5}\right)$. An example of $F^{R}$ is illustrated in [5, Fig. 2]. Equation (17) provides a nonlinear model and will be used for control system design and analysis in Sections III-V.

\section{Motion Stability ANalysis With Static FEEDFORWARD CONTROL}

Fig. 3 shows an illustrative simulation of heave/pitch motion of the planing hull, where porpoising can be clearly observed. Due to the nonlinear nature of the restoring force in (17), setting the transom flap at different static deflection positions will not only shift the equilibrium running attitude $\left(z_{0}, \tau_{0}\right)$, but also change the motion response of the planing vessel around the equilibrium. Effects of the preset static deflection of the transom flap on the heave/pitch motion of the planing vessel are analyzed in this section.

\section{A. Equilibrium Running Attitude}

The equilibrium running attitude of the craft, $\left(z_{0}, \tau_{0}\right)$, can be determined by setting $\dot{x}=0$ and $\delta=\delta_{0}=$ const. in (17). The planing hull studied by Troesch [4] is used here, with $l \mathrm{cg} / B$ being maintained at 1.95 and $C_{\Delta}$ at 1.47 , where $C_{\Delta}$ is 

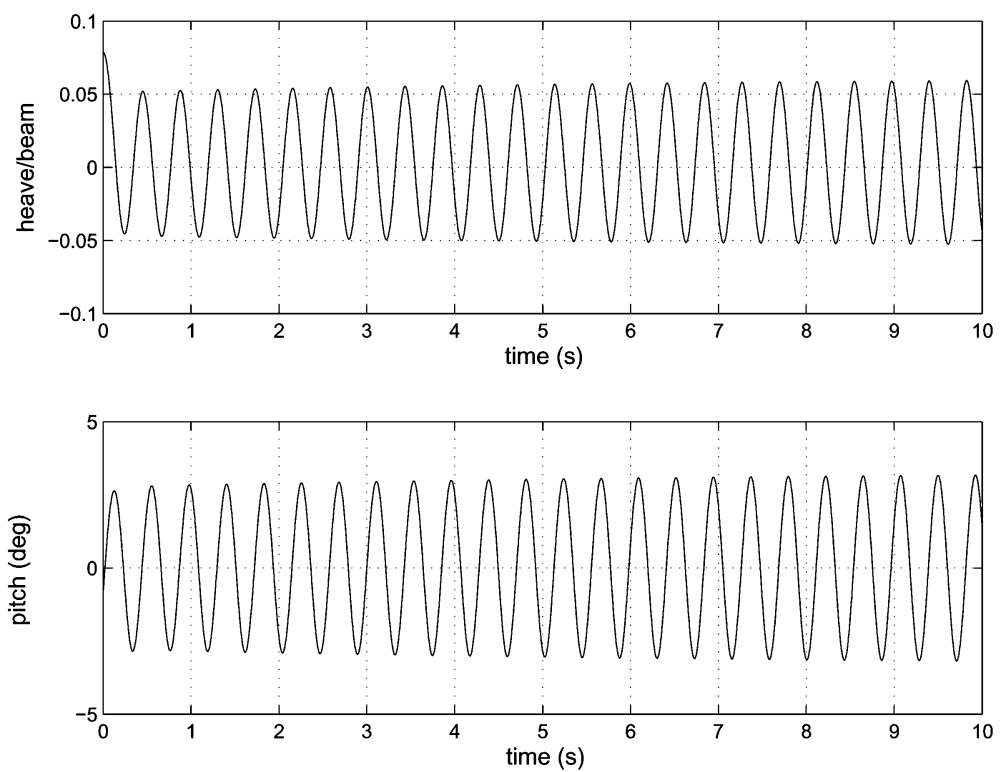

Fig. 3. Heave/pitch motion of the planing vessel when $C_{v}=5.1$ and $\delta=0$.
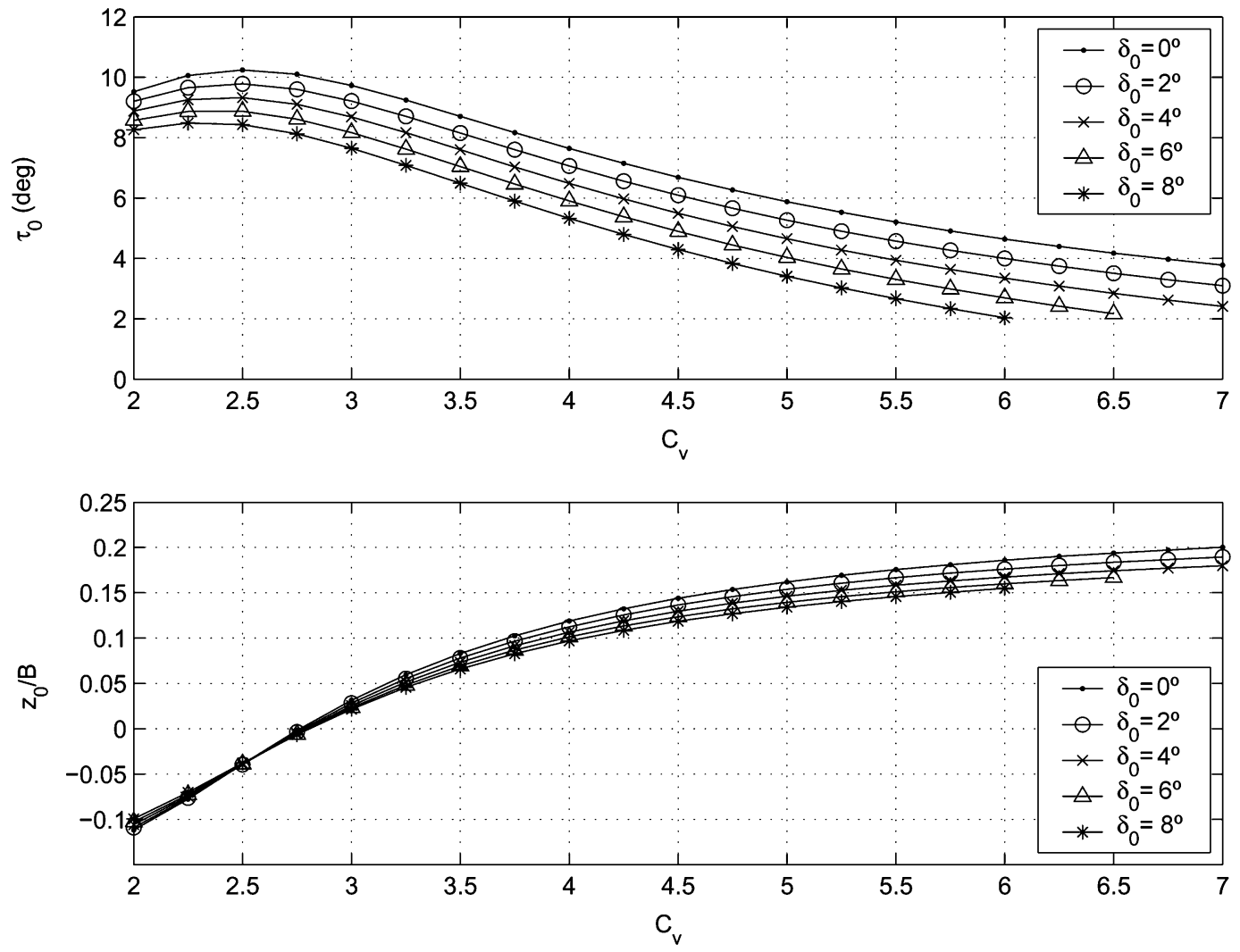

Fig. 4. Equilibrium running attitude.

the beam loading coefficient. A full-span transom flap with the chord length of $0.1 B$ is used to control the craft's motion.

Fig. 4 shows the equilibrium running attitude of the planing craft at different $C_{v}$ and $\delta_{0}$. As Savitsky's method is applicable for trim angles greater than $2^{\circ}$ [3], results with $\tau_{0}<2^{\circ}$ are discarded.

As $\delta_{0}$ increases, the trim angle $\tau_{0}$ decreases at a given forward speed. Larger $\delta_{0}$ increases $z_{0}$ at lower speeds while decreasing $z_{0}$ at higher speeds, but the sensitivity is rather small.
Given $\delta_{0}$, as $C_{v}$ increases, the trim angle $\tau_{0}$ increases before it decreases at higher speeds. A peak of $\tau_{0}$ appears between $C_{v}=2$ and 3 . The increase of $z_{0}$ as $C_{v}$ increases suggests stronger planing effect at higher forward speeds. The trend of the equilibrium running attitude in Fig. 4 can be verified by the experimental results shown in [12].

The equilibrium running attitude obtained here is expected to be close to the actual planing craft running in the calm water. In [5], it is assumed that there exists sufficient mechanism to main- 


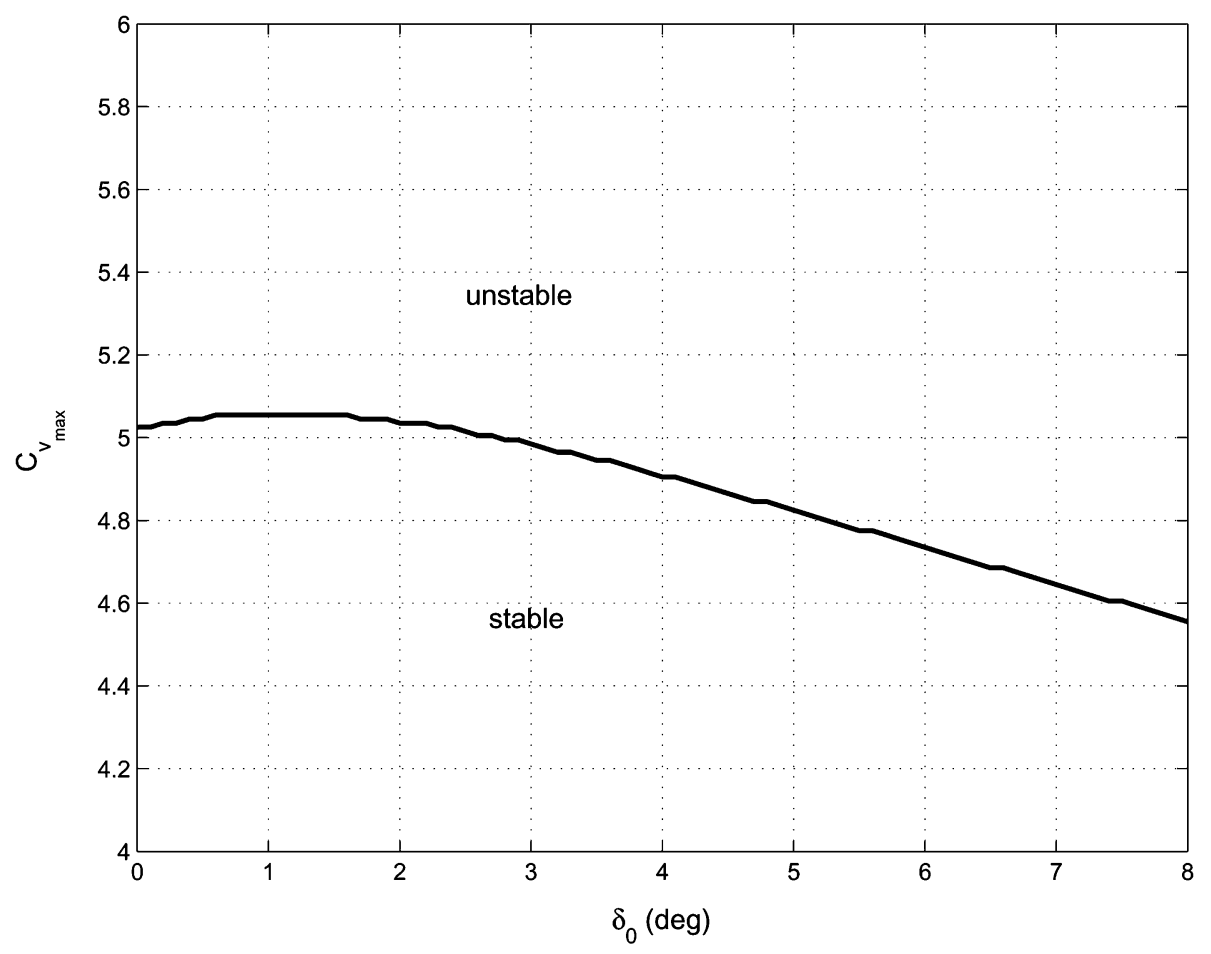

Fig. 5. Maximum speed coefficient where the stability of the planing vessel can be maintained.

tain the boat at any attitude. The equilibrium running attitude is given a priori in their analysis. In towing tank experiments, this assumption may be achieved with proper towing mechanisms. In practice, however, the planing hull, when free in the open water, has no mechanism to maintain arbitrary equilibrium running attitude, even with the controllable transom flap. In our work, no extra external mechanism is assumed to obtain a prescribed equilibrium condition. The equilibrium running attitude is calculated based on the forces induced by the boat motion and the transom flap themselves.

\section{B. Motion Characteristics}

Fig. 5 shows the maximum forward speed coefficient $C_{v_{\max }}$ that the planing hull can maintain without inducing porpoising for different static deflections of the transom flap. Although a small flap deflection can increase the maximum stable speed by a small increment in Fig. 5 (for example, $\delta_{0}=1^{\circ}$ increases $C_{v_{\max }}$ from 5.02 to 5.05), larger deflections reduce the stable operating speed range of the boat. Setting $\delta_{0}=8^{\circ}$ lowers $C_{v_{\max }}$ down to 4.55 . For speeds with $C_{v}>5.06$, the static transom flap is unable to stabilize the heave/pitch motion of the vessel. Therefore, as far as the vertical-plane motion stability is concerned, the static transom flap actually contributes very little to improve the performance of the planing vessel, except that it reduces the amplitude of the porpoising in some cases, as observed in our simulations. In fact, we could conclude that it even aggravates the problem by inducing the onset of porpoising at lower speed. ${ }^{1}$ To extend the maximum stable operating speed range without

${ }^{1}$ While the negative effect of the preset static transom flap on the vessel's stability is verified for the boat considered in this paper, we should point out that, in general, these effects are problem and design specific, and different design parameters could lead to different conclusions. redesigning the hull, dynamic feedback stabilization through the controllable transom flap is pursued in Section IV.

Fig. 6 shows the steady-state motion of the planing vessel in the state-space. By simulating the model from different initial perturbations, the boat's steady-state motions result in the same pattern as shown in Fig. 6, which suggests that it is initial condition independent and the motion of porpoising under this operating condition is basically a stable limit cycle.

It should be noted that Savitsky's method is applicable within certain conditions, such as $\lambda \leq 4$ and $2^{\circ} \leq \tau \leq 15^{\circ}$ [3]. In our simulation, these limits are often violated and, therefore, the results have to be discarded. To extend the results to larger motions, it is important to modify the empirical relations and expand the applicable range. This will be a topic of future research.

\section{STABILIZATION BASED ON FeEdBack LINEARIZATION METHOD}

A nonlinear controller based on the feedback linearization method is developed in this section to stabilize the planing craft at high speeds. The control scheme is illustrated in Fig. 7, where $u_{\delta}=k(x)$ is the feedback control law to be designed. The deflection of the transom flap $\delta$ is decomposed into two parts: $\delta=\delta_{0}+u_{\delta}$, where $\delta_{0}$ is the preset nominal deflection that determines the equilibrium running attitude of the planing craft, and $u_{\delta}$ is the relative deflection about $\delta_{0}$ as a feedback control input.

Given $\delta_{0}$, the model in (17) can be rewritten in the following form:

$$
\dot{x}=\hat{f}(x)+b u_{\delta}
$$

where $\hat{f}(x)=f\left(z_{0}, \tau_{0} ; x\right)+b \delta_{0} .\left(z_{0}, \tau_{0}\right)$ is the equilibrium running attitude of the vessel corresponding to the nominal flap 

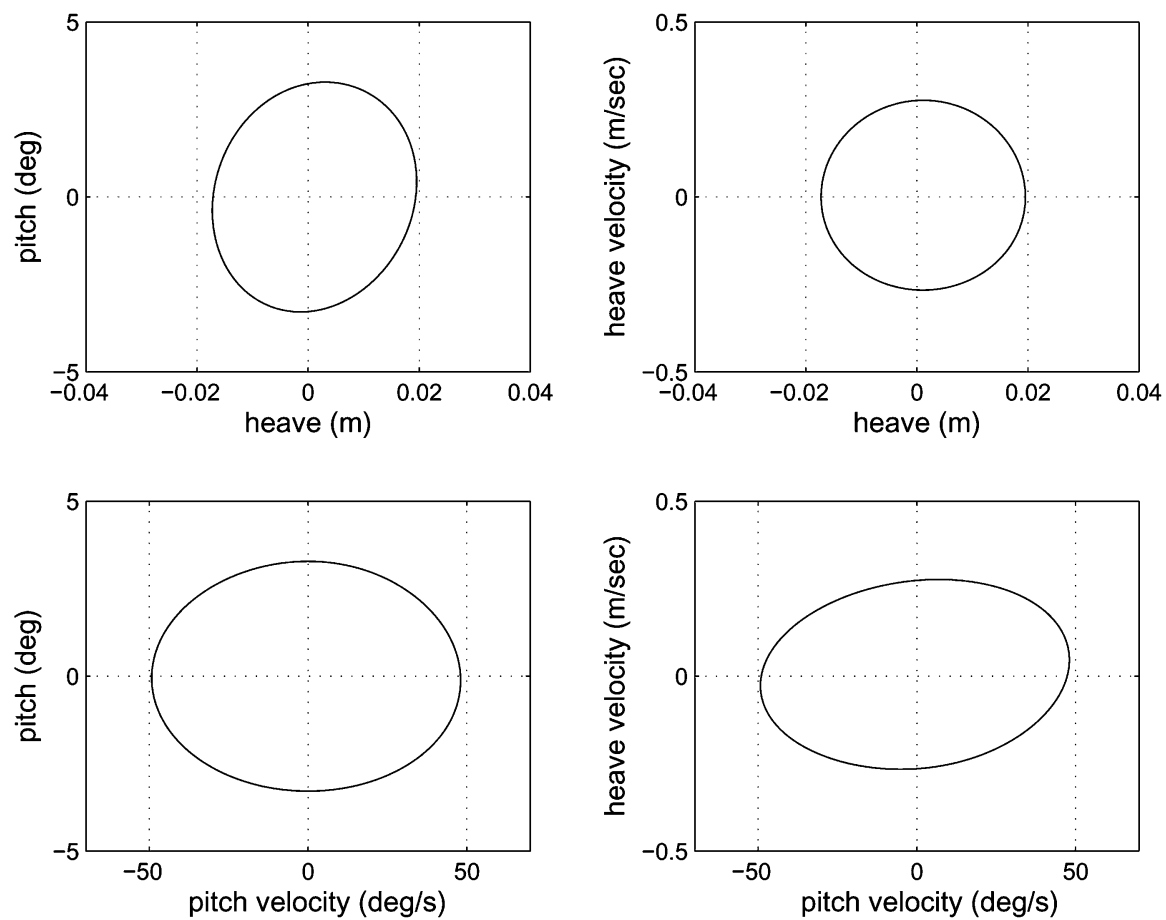

Fig. 6. Steady-state motion trajectory in the state-space. $C_{v}=5.1$ and $\delta_{0}=0$.

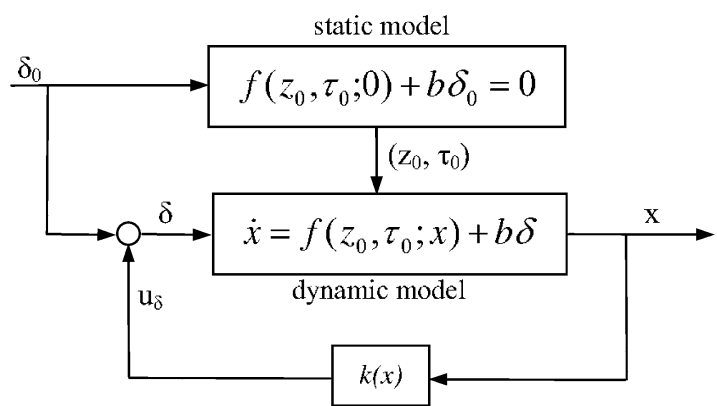

Fig. 7. Control loop of the system.

deflection $\delta_{0}$. The objective is to design a feedback control law $u_{\delta}=k(x)$, using feedback linearization to stabilize the system of planing vessels described by the model of (18) at the equilibrium of $x=0$.

For convenience, the following notations are defined and will be used in the sequel:

$$
\begin{aligned}
{\left[\begin{array}{ll}
\alpha_{1} & \alpha_{2} \\
\alpha_{3} & \alpha_{4}
\end{array}\right] } & :=-A^{-1} B \\
{\left[\begin{array}{l}
\gamma_{1} \\
\gamma_{2}
\end{array}\right] } & :=A^{-1}\left[\begin{array}{l}
d_{3} \\
d_{5}
\end{array}\right] \\
{\left[\begin{array}{l}
\hat{\theta}_{1}\left(x_{1}, x_{2}\right) \\
\hat{\theta}_{2}\left(x_{1}, x_{2}\right)
\end{array}\right] } & :=A^{-1} F^{R}\left(z_{0}, \tau_{0} ; x_{1}, x_{2}\right)+\left[\begin{array}{l}
\gamma_{1} \\
\gamma_{2}
\end{array}\right] \delta_{0} .
\end{aligned}
$$

\section{A. Feedback Linearizability}

If the system in (18) can be transformed into a linear form by applying a state transformation and state feedback, then, stabilizing controllers can be easily designed based on this linear form. For the system model under consideration, however, we have the following.
Proposition 4.1: The model of planing hulls described in (18) is not fully feedback linearizable.

To verify the proposition, we need the following theorem which gives the sufficient and necessary conditions for the system feedback linearizability [13].

Theorem 4.1: The system $\dot{x}=\varphi(x)+g(x) u$, where $x$ is $n$-dimensional, is fully feedback linearizable near $x^{0}$ if and only if the following conditions are satisfied:

i) the matrix $P:=\left[g\left(x^{0}\right), a d_{\varphi} g\left(x^{0}\right), \ldots, a d_{\varphi}^{n-1} g\left(x^{0}\right)\right]$ has rank $n$, where $a d_{\varphi} g(x):=(\partial g / \partial x) \varphi(x)-$ $(\partial \varphi / \partial x) g(x)$

ii) the distribution $\Omega:=\operatorname{span}\left\{g, a d_{\varphi} g, \ldots, a d_{\varphi}^{n-2} g\right\}$ is involutive (i.e., $\forall \omega_{1}, \omega_{2} \in \Omega \Rightarrow a d_{\omega_{1}} \omega_{2} \in \Omega$ ) near $x^{0}$.

Proof of Proposition 4.1: We will show that the model given by (18) is not fully feedback linearizable by using Theorem 4.1 and proving that $\Omega$ is not involutive. For the boat system described by (18), $n=4, \varphi(x)=\hat{f}(x), g(x)=b$, $x^{0}=0$. Thus, using the definition of $\Omega$, we obtain

$$
\begin{aligned}
\Omega & =\operatorname{span}\left\{b, a d_{\hat{f}} b, a d_{\hat{f}}^{2} b\right\} \\
& =\operatorname{span}\left\{\begin{array}{ccc}
0 & -\gamma_{1} & \gamma_{1} \alpha_{1}+\gamma_{2} \alpha_{2} \\
0 & -\gamma_{2} & \gamma_{1} \alpha_{3}+\gamma_{2} \alpha_{4} \\
\gamma_{1} & -\gamma_{1} \alpha_{1}-\gamma_{2} \alpha_{2} & \phi_{1}\left(x_{1}, x_{2}\right) \\
\gamma_{2} & -\gamma_{1} \alpha_{3}-\gamma_{2} \alpha_{4} & \phi_{2}\left(x_{1}, x_{2}\right)
\end{array}\right\}
\end{aligned}
$$

where

$$
\begin{aligned}
\phi_{1}\left(x_{1}, x_{2}\right):= & \gamma_{1} \frac{\partial \hat{\theta}_{1}}{\partial x_{1}}+\gamma_{2} \frac{\partial \hat{\theta}_{1}}{\partial x_{2}}+\alpha_{1}\left(\gamma_{1} \alpha_{1}+\gamma_{2} \alpha_{2}\right) \\
& +\alpha_{2}\left(\gamma_{1} \alpha_{3}+\gamma_{2} \alpha_{4}\right) \\
\phi_{2}\left(x_{1}, x_{2}\right):= & \gamma_{1} \frac{\partial \hat{\theta}_{2}}{\partial x_{1}}+\gamma_{2} \frac{\partial \hat{\theta}_{2}}{\partial x_{2}}+\alpha_{3}\left(\gamma_{1} \alpha_{1}+\gamma_{2} \alpha_{2}\right) \\
& +\alpha_{4}\left(\gamma_{1} \alpha_{3}+\gamma_{2} \alpha_{4}\right) .
\end{aligned}
$$


If $\Omega$ is involutive, we should have $a d_{a d_{\hat{f}} b}\left(a d_{\hat{f}}^{2} b\right) \in \Omega$, i.e., $a d_{a d_{\hat{f}} b}\left(a d_{\hat{f}}^{2} b\right)$ can be expressed as a linear combination of $b$, $a d_{\hat{f}} b$, and $a d_{\hat{f}}^{2} b$. However, note that

$a d_{a d_{\hat{f}} b}\left(a d_{\hat{f}}^{2} b\right)=\left[\begin{array}{c}0 \\ 0 \\ -\left.\gamma_{1}^{2} \frac{\partial^{2} \hat{\theta}_{1}}{\partial x_{1}^{2}}\right|_{0}-\left.2 \gamma_{1} \gamma_{2} \frac{\partial^{2} \hat{\theta}_{1}}{\partial x_{1} \partial x_{2}}\right|_{0}-\left.\gamma_{2}^{2} \frac{\partial^{2} \hat{\theta}_{1}}{\partial x_{2}^{2}}\right|_{0} \\ -\left.\gamma_{1}^{2} \frac{\partial^{2} \hat{\theta}_{2}}{\partial x_{1}^{2}}\right|_{0}-\left.2 \gamma_{1} \gamma_{2} \frac{\partial^{2} \hat{\theta}_{2}}{\partial x_{1} \partial x_{2}}\right|_{0}-\left.\gamma_{2}^{2} \frac{\partial^{2} \hat{\theta}_{2}}{\partial x_{2}^{2}}\right|_{0}\end{array}\right]$

and the matrix $\left[b, a d_{\hat{f}} b, a d_{\hat{f}}^{2} b, a d_{a d_{\hat{f}} b}\left(a d_{\hat{f}}^{2} b\right)\right]$ has full rank around $x^{0}=0$; we conclude that $a d_{a d_{\hat{f}}}\left(a d_{\hat{f}}^{2} b\right)$ does not belong to $\Omega$. Hence, $\Omega$ is not involutive. According to Theorem 4.1, our model is not fully feedback linearizable.

We now turn to search for a state transformation which can partially linearize the system.

Proposition 4.2: For the planing vessel system described by (18), there exists a state transformation $z=\left[z_{1}, z_{2}, z_{3}, z_{4}\right]^{T}=$ $\Phi(x)$, such that the system can be transformed into the following form:

$$
\begin{aligned}
\dot{\hat{z}} & =E \hat{z}+F v \\
\dot{z}_{4} & =q(z)
\end{aligned}
$$

where

$$
\hat{z}=\left[\begin{array}{l}
z_{1} \\
z_{2} \\
z_{3}
\end{array}\right] \quad E=\left[\begin{array}{lll}
0 & 1 & 0 \\
0 & 0 & 1 \\
0 & 0 & 0
\end{array}\right] \quad F=\left[\begin{array}{l}
0 \\
0 \\
1
\end{array}\right]
$$

and

$$
v=g_{1}(x)+g_{2}(x) u_{\delta}
$$

for some functions $g_{1}(x), g_{2}(x)$ with $g_{2}(0) \neq 0$.

Proof: Consider the following virtual output function:

$$
h(x)=-\gamma_{2} x_{1}+\gamma_{1} x_{2} .
$$

Define a state transformation $z=\Phi(x)$ based on $h(x)$ as follows:

$$
\left[\begin{array}{c}
z_{1} \\
z_{2} \\
z_{3} \\
z_{4}
\end{array}\right]=\Phi(x):=\left[\begin{array}{c}
h(x) \\
L_{\hat{f}} h(x) \\
L_{\hat{f}}^{2} h(x) \\
x_{1}
\end{array}\right]
$$

where $L_{\hat{f}}(\cdot)$ is the Lie derivative along $\hat{f}(x)$, i.e., $L_{\hat{f}}(\cdot)=$ $(\partial(\cdot) / \partial x) \hat{f}(x)$. Substitute $h(x)$ into (22), we have

$$
\Phi(x)=\left[\begin{array}{c}
-\gamma_{2} x_{1}+\gamma_{1} x_{2} \\
-\gamma_{2} x_{3}+\gamma_{1} x_{4} \\
\beta_{1} x_{3}+\beta_{2} x_{4}+\gamma_{1} \hat{\theta}_{2}\left(x_{1}, x_{2}\right)-\gamma_{2} \hat{\theta}_{1}\left(x_{1}, x_{2}\right) \\
x_{1}
\end{array}\right]
$$

where $\beta_{1}=\left(\gamma_{1} \alpha_{3}-\gamma_{2} \alpha_{1}\right), \beta_{2}=\left(\gamma_{1} \alpha_{4}-\gamma_{2} \alpha_{2}\right)$. Then, the system in (18) can be transformed into

$$
\begin{aligned}
\dot{\hat{z}} & =E \hat{z}+F\left[L_{\hat{f}}^{3} h(x)+L_{b} L_{\hat{f}}^{2} h(x) u_{\delta}\right] \\
\dot{z}_{4} & =x_{3} .
\end{aligned}
$$

It can be shown that the Jacobion matrix of $\Phi(x)$ is nonsingular at $x=0$. Hence, the transformation defined in (22) is a diffeomorphism and its inverse mapping $x=\Phi^{-1}(z)$ exists.

Let

$$
\begin{aligned}
v & =L_{\hat{f}}^{3} h(x)+L_{b} L_{\hat{f}}^{2} h(x) u_{\delta} \\
q(z) & =x_{3}
\end{aligned}
$$

and express $x_{3}$ in (26) with $z$ using the inverse mapping $x=$ $\Phi^{-1}(z)$ we obtain the state-space model in the new coordinates in the form given in Proposition 4.2.

Apparently, $\Phi(0)=0$ and thus $z=0$ is the corresponding equilibrium of the system in the new coordinates.

Viewing $v$ as the new control input, the model described in (19) and (20) decomposes the system into two parts in the new coordinates: A linear part described by (19) and a nonlinear internal dynamics by (20). If $v$ is designed to stabilize the whole system including the internal dynamics, the stabilizing control input $u_{\delta}$ in the original coordinate of $x$ can be obtained by

$$
u_{\delta}(x)=\frac{v-L_{\hat{f}}^{3} h(x)}{L_{b} L_{\hat{f}}^{2} h(x)}
$$

where $L_{b} L_{\hat{f}}^{2} h(x)=\beta_{1} \gamma_{1}+\beta_{2} \gamma_{2}>0$ for the speed range of $C_{v}=2.0 \sim 7.0$ considered in our study.

\section{B. Zero Dynamics and Local Stability of System}

While linear control theory can be applied to design the control input $v$ to stabilize the linear part described by (19), the stability of the internal dynamics in (20) is required to establish the stability for the whole system of the planing vessel. The effect of the internal dynamics on the system stability can be analyzed through the so-called zero dynamics.

Setting $z_{1}=z_{2}=z_{3}=0$ in the internal dynamics of (20) results in

$$
\dot{z}_{4}=q\left(\left[0,0,0, z_{4}\right]^{T}\right)
$$

which corresponds to the zero dynamics. The following lemma can be derived for the system stability from the feedback linearization theory [13], and the proof is omitted here.

Lemma 4.1: Suppose the equilibrium $z_{4}=0$ of the zero dynamics (28) is locally asymptotically stable and $v=-K \hat{z}$ where $K$ is designed such that $(E-F K)$ is Hurwitz. ${ }^{2}$ Then the feedback law in (27) locally asymptotically stabilizes the original system (18) at the equilibrium of $x=0$.

From Lemma 4.1, the local stability of the zero dynamics is critical to establish the local stability of the whole system using 


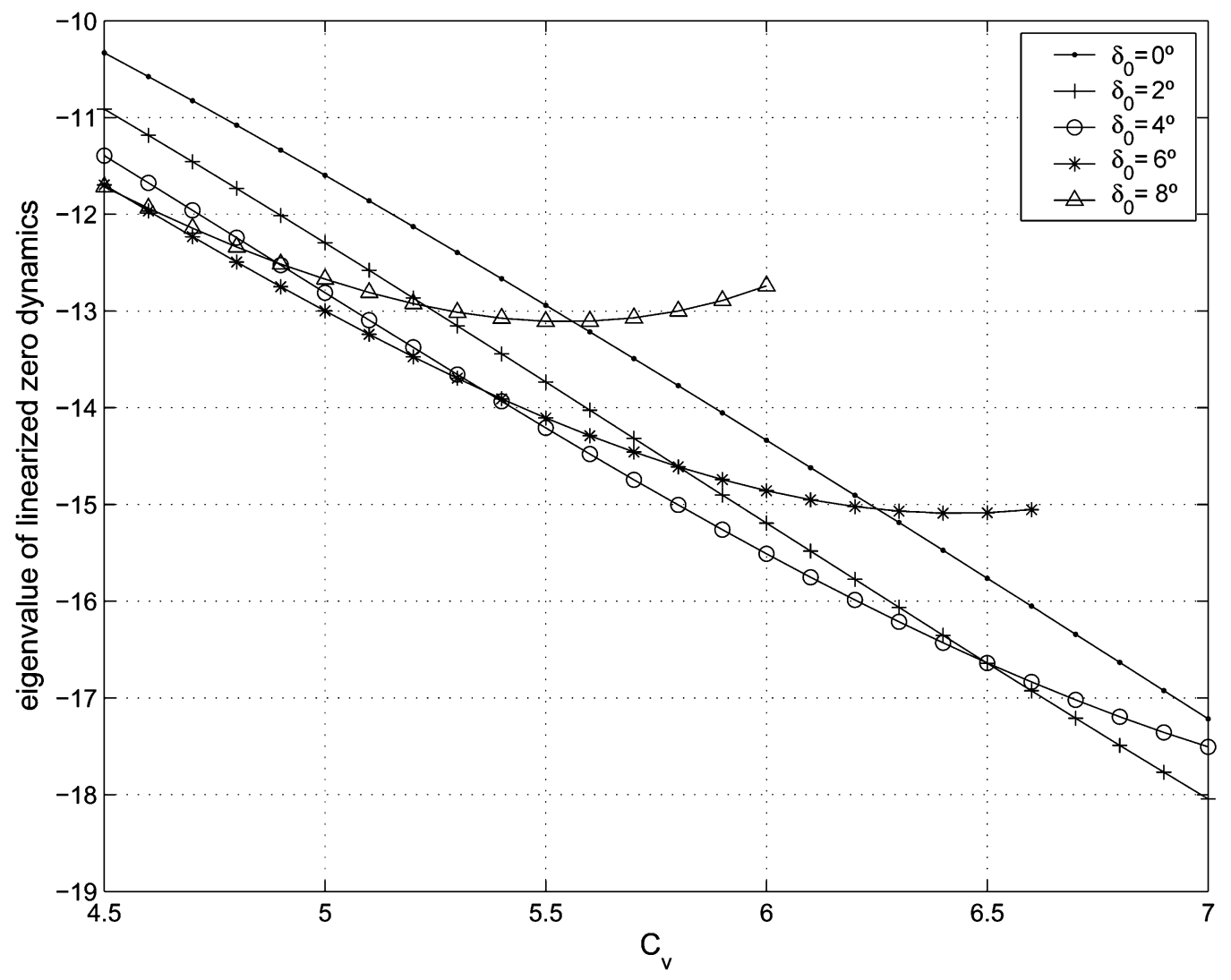

Fig. 8. Eigenvalue of linearized zero dynamics.

the feedback linearization approach based on (19) and (20). The linearized zero dynamics at $z_{4}=0$ is

$$
\dot{z}_{4}=\left.\frac{\partial q\left(\left[0,0,0, z_{4}\right]^{T}\right)}{\partial z_{4}}\right|_{z_{4}=0} z_{4} .
$$

Fig. 8 shows that the eigenvalue of the linearized zero dynamics of the planing craft is negative for different operating conditions of $C_{v}$ and $\delta_{0}$, and therefore, the zero dynamics are locally asymptotically stable at $z_{4}=0$. The local stability of the composite system is then established by Lemma 4.1, when $v=-K \hat{z}$ is chosen such that $(E-F K)$ is Hurwitz.

\section{Simulations and Discussions}

The feedback stabilizing control law in the new coordinate $v=-K \hat{z}$ can be designed by using the pole placement method. Fig. 9 shows two examples where the planing vessel is stabilized and porpoising eliminated. The poles of $(E-F K)$ are placed at $[-4+4 j,-4-4 j,-8]^{T}$ by properly chosen $K$.

When there are uncertainties in the model, which is inevitable for this application, the robustness of the controller due to feedback linearization is always a concern since such controller requires "exact" cancellation of nonlinearities. For the planing vessel, the uncertainties in the model, whether associated with $A, B, F^{R}$, or $F^{F}$, will manifest as extra terms in the state equation of (23) and (24) for $\dot{z}_{3}$ and $\dot{z}_{4}$. Note that the uncertainty term in (23) is the so-called "matched" uncertainty as it enters the system through the same channel as the control input. The matched uncertainties are usually considered as not destructive, since many linear/nonlinear control design schemes are available to address them and to enforce system robustness. For the uncertainties that appear in (24), as long as they do not change the property of $\left.\left(\partial q\left(\left[0,0,0, z_{4}\right]^{T}\right) / \partial z_{4}\right)\right|_{z_{4}=0}<0$, the stability and robustness of the equilibrium for the closed loop system will not be affected.

\section{ANALYSIS ON THE REGION OF ATtRACTION}

The controller designed in the previous section establishes local asymptotic stability for the planing vessel at high speeds. But the analysis does not specify the region of attraction. In other words, the results of Section IV establish that if the initial condition is "sufficiently" close to the equilibrium, then a stable motion is guaranteed. However, a quantitative measure of sufficient closeness is not specified. For practical purpose, it is of interest to investigate conditions under which the system can be led to the equilibrium at the origin. This section is devoted to assessing the region of attraction for the equilibrium point and to define the safe operating range of the high-speed planing boat.

Given that the state $\hat{z}$, consisting of $z_{1}, z_{2}$ and $z_{3}$, is governed by a linear subsystem, the region of attraction for the stabilized equilibrium point $z=0$ of the system (19) and (20), with $v=$ $-K \hat{z}$ and $(E-F K)$ being Hurwitz, is primarily dictated by the nonlinear internal dynamics defined by (20). Note that (20) has the form of

$$
\dot{z}_{4}=\tilde{f}\left(z_{1}, z_{4}\right)+s_{1} z_{2}+s_{2} z_{3}
$$


(a)
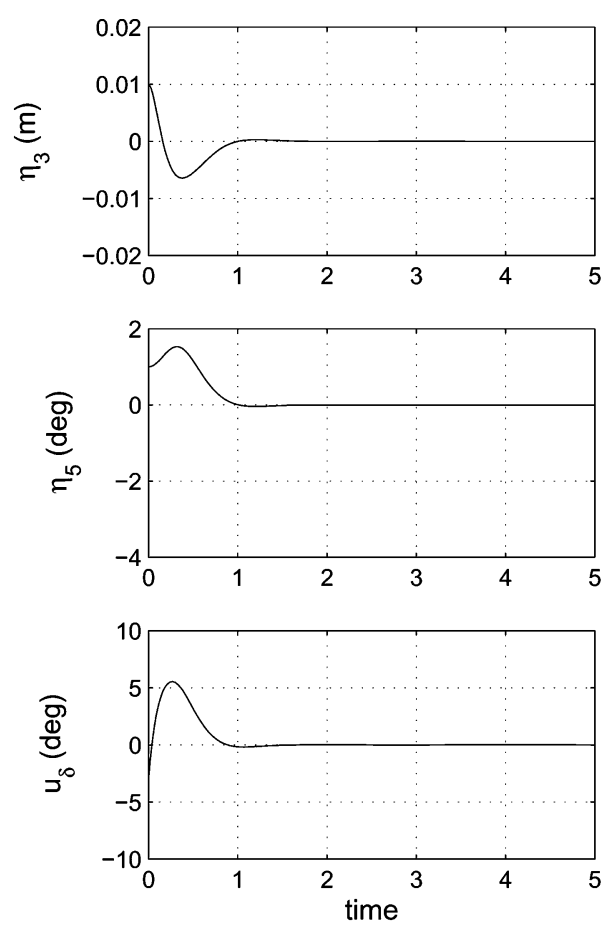

(b)
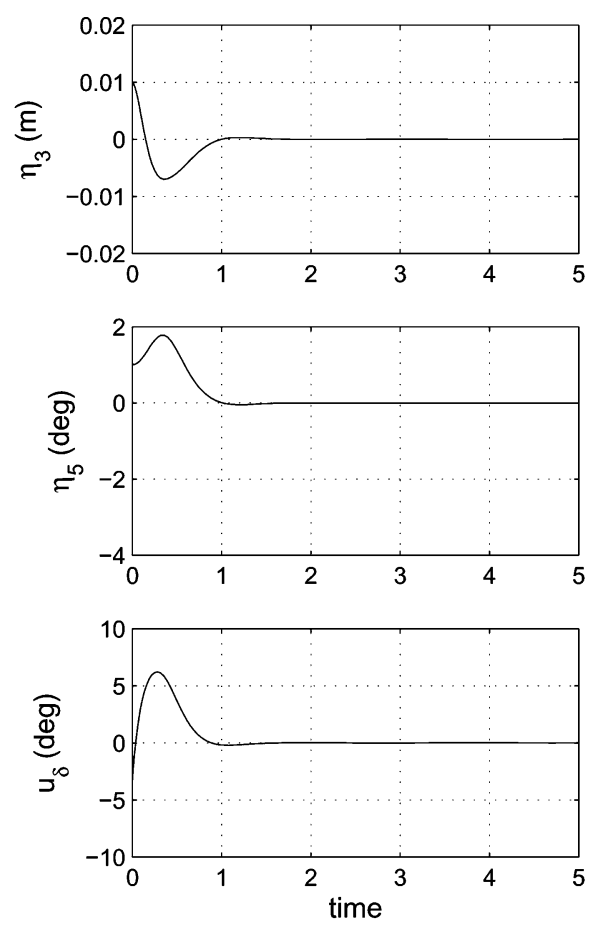

Fig. 9. Response and control input of porpoising stabilization. $\delta_{0}=3^{\circ}$ and $x(0)=\left[0.01 \mathrm{~m}, 1^{\circ}, 0,0\right]^{T}$. (a) $C_{v}=5.5$. (b) $C_{v}=6.0$.

where

$$
\begin{aligned}
\tilde{f}\left(z_{1}, z_{4}\right) & =-\frac{1}{\beta}\left[\gamma_{1}^{2} \hat{\theta}_{2}\left(z_{4}, \frac{1}{\gamma_{1}}\left(z_{1}+\gamma_{2} z_{4}\right)\right)\right. \\
\left.-\gamma_{1} \gamma_{2} \hat{\theta}_{1}\left(z_{4}, \frac{1}{\gamma_{1}}\left(z_{1}+\gamma_{2} z_{4}\right)\right)\right] & \\
s_{1} & =-\frac{\beta_{1}}{\beta} \\
s_{2} & =\frac{\gamma_{1}}{\beta} \\
\beta & =\gamma_{1}\left(\gamma_{1} \alpha_{3}-\gamma_{2} \alpha_{1}\right)+\gamma_{2}\left(\gamma_{1} \alpha_{4}-\gamma_{2} \alpha_{2}\right) .
\end{aligned}
$$

By defining

$$
\begin{aligned}
\tilde{f}_{\hat{z}}(\hat{z}) & =\tilde{f}\left(z_{1}, 0\right)+s_{1} z_{2}+s_{2} z_{3} \\
\tilde{f}_{z_{4}}\left(z_{1}, z_{4}\right) & =\tilde{f}\left(z_{1}, z_{4}\right)-\tilde{f}\left(z_{1}, 0\right)
\end{aligned}
$$

we can rewrite (20) in the form of

$$
\dot{z}_{4}=\tilde{f}_{z_{4}}\left(z_{1}, z_{4}\right)+\tilde{f}_{\hat{z}}(\hat{z}) .
$$

We then have the following proposition concerning the stability of the planing craft motion with different initial conditions.

Proposition 5.1: If $\tilde{f}_{\hat{z}}$ and $\tilde{f}_{z_{4}}$ given by (31) and (32) satisfy the following conditions:

$$
\left|\tilde{f}_{\hat{z}}(\hat{z})\right| \leq k_{1}|\hat{z}|
$$

and

$$
z_{4} \tilde{f}_{z_{4}}\left(z_{1}, z_{4}\right) \leq-k_{2} z_{4}^{2}
$$

for some constants $k_{1}, k_{2}>0$, and the control law $v=-K \hat{z}$ is designed such that $(E-F K)$ is Hurwitz, then

$$
z(t) \rightarrow 0 \text { as } t \rightarrow+\infty \text {. }
$$

Proof: Since $(E-F K)$ is designed to be Hurwitz, there exist positive constants $p$ and $q$ such that

$$
k_{1}|\hat{z}| \leq p e^{-q t}
$$

where $q$ is dependent upon the controller gain $K$ and $p$ on $k_{1}$, $K$, and the initial condition $\hat{z}(0)$. Thus, defining a nonnegative function $V\left(z_{4}\right):=z_{4}^{2}$, we have

$$
\begin{aligned}
\dot{V} & =2 z_{4}\left[\tilde{f}_{z_{4}}\left(z_{1}, z_{4}\right)+\tilde{f}_{\hat{z}}(\hat{z})\right] \\
& \leq-2 k_{2} z_{4}^{2}+2\left|z_{4}\right| k_{1}|\hat{z}| \\
& \leq-2 k_{2} z_{4}^{2}+2\left|z_{4}\right| p e^{-q t} \\
& =-2 k_{2} V+2 \sqrt{V} p e^{-q t} .
\end{aligned}
$$

Let $\tilde{V}:=\sqrt{V}=\left|z_{4}\right|$, from (37), we conclude that

$$
\dot{\tilde{V}} \leq-k_{2} \tilde{V}+p e^{-q t} \text {. }
$$

Using [15, Lemma 3.2.4], we can show that (38) implies

$$
\tilde{V}(t) \leq \tilde{V}(0) e^{-k_{2} t}-\frac{p}{q-k_{2}}\left(e^{-q t}-e^{-k_{2} t}\right) .
$$

Therefore, $\left|z_{4}(t)\right|=\tilde{V}(t) \rightarrow 0$ as $t \rightarrow+\infty$ follows by noting that $k_{2}, q>0$. Note that $(E-F K)$ being Hurwitz implies that $\hat{z}(t) \rightarrow 0$ as $t \rightarrow+\infty$; we conclude that

$$
z(t)=\left[\begin{array}{c}
\hat{z}(t) \\
z_{4}(t)
\end{array}\right] \rightarrow 0
$$

as $t \rightarrow+\infty$. 


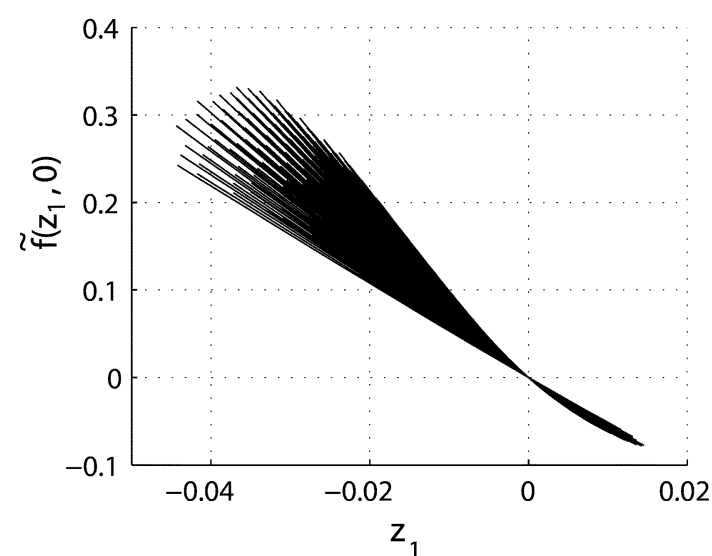

(a)

Fig. 10. Plots of $\tilde{f}\left(z_{1}, 0\right)$ and $\tilde{f}_{z_{4}}\left(z_{1}, z_{4}\right)$ in the model applicable range.

For the planing vessel model described in Section II, conditions (34) and (35) are satisfied for the states within the range covered by Savitsky's empirical methods. To validate (34), we note that since other two terms in $\tilde{f}_{\hat{z}}$ are linear [see (31)], the Lipschitz condition for $\tilde{f}_{\hat{z}}$ is satisfied if $\tilde{f}\left(z_{1}, 0\right)$ is Lipschitz in $z_{1}$. The latter is true by examining Fig. 10(a), which plots $\tilde{f}\left(z_{1}, 0\right)$ as a function of $z_{1}$ for all values of $z_{1}$ that are within the applicable range of the model, while $C_{v}$ and $\delta$ are varied within the range of $5 \leq C_{v} \leq 7,0 \leq \delta \leq 8^{\circ}$ to reflect the operating range of the planing vessel and the static deflection of the transom flap.

For condition (35), we plot $\tilde{f}_{z_{4}}$ as a function of $z_{4}$ for all possible $\left(z_{1}, z_{4}\right)$ combinations covered by the Savitsky's method, as given in Fig. 10(b). It is clear that (35) is also satisfied for the model of the planing vessel.

The above analysis indicates that, if the initial condition $z(0)$ and the controller gain $K$ are chosen such that the motion trajectory of the planing boat remains in the applicable range of the Savitsky's method, then motion stability is guaranteed and porpoising is eliminated.

\section{CONCLUSION}

A nonlinear controller based on the feedback linearization method is designed to enhance the vertical-plane stability of the high-speed planing vessel using a controllable transom flap. A state transformation is constructed to transform the system into a partially linear form, and local asymptotic stability is obtained by verifying the local stability of zero dynamics. We also show that the proposed stabilizing controller guarantees motion stability if the boat's movement remains in the applicable range of the model.

The modeling and control results described in this paper provide a baseline for many future research topics. On the experimental side, we plan to develop a scaled model to validate the control design in a towing tank facility at the University of Michigan, Ann Arbor. The mathematical model presented in this paper will be further extended for the planing vessels running in waves. Coordinated controls for the longitudinal and roll motion with vertical motions will also be investigated with augmented control actuation.

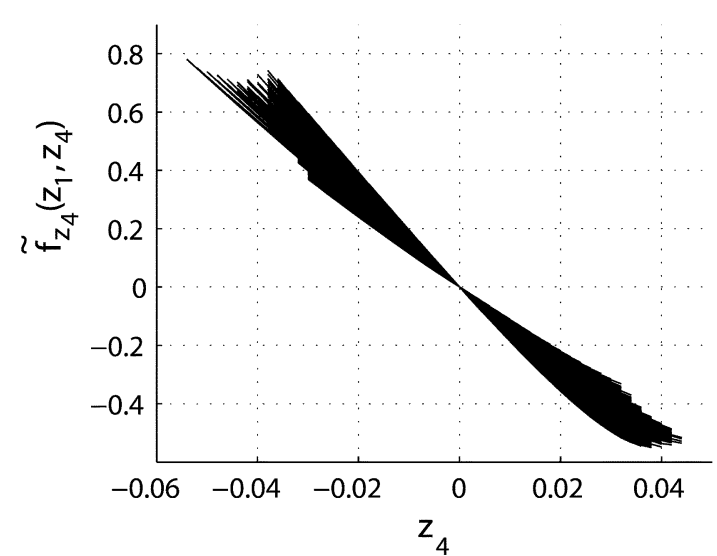

(b)

\section{ACKNOWLEDGMENT}

The authors would like to thank Prof. A. W. Troesch and Dr. B. R. Savander of the Naval Architecture and Marine Engineering Department, and Prof. N. H. McClamroch of the Aerospace Engineering Department, all from the University of Michigan, for discussions regarding the modeling and control design of this work.

\section{REFERENCES}

[1] D. L. Blount and L. T. Codega, "Dynamic stability of planing boats," Mar. Tech., vol. 29, no. 1, pp. 4-12, Jan. 1992.

[2] Y. Ikeda et al., "Stability of high speed craft," in Contemporary Ideas on Ship Stability, D. Vassalos et al., Eds. New York: Elsevier Science Ltd , 2000, pp. 401-409.

[3] D. Savitsky, "Hydrodynamic design of planing hulls," Mar. Tech., vol. 1, no. 1, pp. 71-95, Oct. 1964.

[4] A. W. Troesch, "On the hydrodynamics of vertically oscillating planing hulls," J. Ship Res., vol. 36, no. 4, pp. 317-331, Dec. 1992.

[5] A. W. Troesch and J. M. Falzarano, "Modern nonlinear dynamical analysis of vertical plane motion of planing hulls," J. Ship Res., vol. 37, no. 3, pp. 189-199, Sep. 1993.

[6] M. Martin, "Theoretical determination of porpoising instability of highspeed planing boats," J. Ship Res., vol. 22, no. 1, pp. 32-53, Mar. 1978.

[7] - "Theoretical prediction of motions of high-speed planing boats in waves," J. Ship Res., vol. 22, no. 3, pp. 140-169, Sep. 1978.

[8] J. D. Hicks, A. W. Troesch, and C. Jiang, "Simulation and nonlinear dynamics analysis of planing hulls," J. Offshore Mech. Arctic Eng., vol. 117, pp. 38-45, Feb. 1995.

[9] J. M. De la Cruz, J. Aranda, J. M. Giron-Sierra, F. Velasco, S. Esteban, J. M. Diaz, and B. de Andres-Toro, "Improving the comfort of a fast ferry," IEEE Control Syst. Mag., vol. 24, no. 2, pp. 47-60, Apr. 2004.

[10] D. Savitsky and P. W. Brown, "Procedures for hydrodynamic evaluation of planing hulls in smooth and rough water," Mar. Tech., vol. 13, no. 4, pp. 381-400, Oct. 1976.

[11] H. Xi and J. Sun, "Vertical plane motion of high speed planing vessels with controllable transom flaps: Modeling and control," in Proc. Int. Federation Automatic Control World Congr. (IFAC), Prague, Czech Republic, Jul., 3-8 2005.

[12] T. Katayama, T. Hinami, and Y. Ikeda, "Longitudinal motion of a super high-speed planing craft in regular head waves," in Proc. 4th Osaka Colloquium on Seakeeping Performance of Ships, 2000, pp. 214-220.

[13] A. Isidori, Nonlinear Control Systems, 3rd ed. New York: SpringerVerlag, 1995.

[14] J. Katz and A. Plotkin, Low-Speed Aerodynamics: From Wing Theory to Panel Methods. New York: McGraw-Hill, 1991.

[15] P. Ioannou and J. Sun, Robust Adaptive Control. Englewood Cliffs, NJ: Prentice-Hall, 1996. 


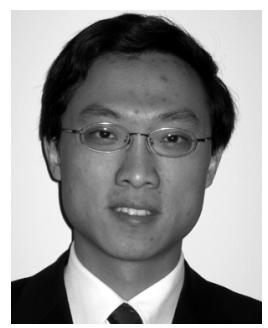

Handa Xi (S'05) received the B.E. and M.E. degrees from Shanghai Jiao Tong University, Shanghai, China, in 1999 and 2002, respectively, both in mechanical engineering. He is currently working toward the Ph.D. degree in the Naval Architecture and Marine Engineering Department, University of Michigan, Ann Arbor

His research interest focuses on dynamic system analysis and control with applications to high-speed vessels and fuel cell systems.

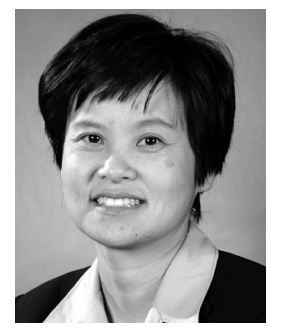

Jing Sun (S'87-M'89-SM'00-F'04) received the B.S. and M.S. degrees from University of Science and Technology of China, Hefei, China, in 1982 and 1984, respectively, and the Ph.D degree from the University of Southern California, Los Angeles, in 1989.

From 1989 to 1993, she was an Assistant Professor in Electrical and Computer Engineering Department, Wayne State University, Detroit, MI. She joined Ford Research Laboratory, Dearborn, MI, in 1993, where she worked in the Powertrain Control Systems Department. After working almost ten years in industry, she came back to academia and joined the faculty of the College of Engineering at University of Michigan, Ann Arbor, in 2003, as an Associate Professor. She holds over 30 U.S. patents and has coauthored a textbook on robust adaptive control. Her research interests include system and control theory and its applications to marine and automotive propulsion systems.

Dr. Sun was one of the three recipients of the 2003 IEEE Control System Technology Award. 